\title{
Pathological Complete Response Following Different Neoadjuvant Treatment Strategies for Locally Advanced Rectal Cancer: A Systematic Review and Meta-analysis
}

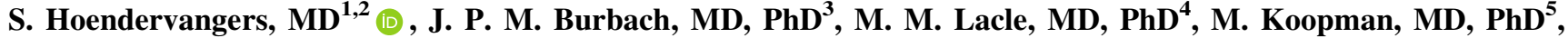 \\ W. M. U. van Grevenstein, MD, $\mathrm{PhD}^{2}$, M. P. W. Intven, $\mathrm{MD}, \mathbf{P h D}^{1}$, and $\mathrm{H}$. M. Verkooijen, $\mathrm{MD}, \mathbf{P h D}^{1}$ \\ ${ }^{1}$ Department of Radiation Oncology, University Medical Center Utrecht, Utrecht, The Netherlands; ${ }^{2}$ Department of \\ Surgery, University Medical Center Utrecht, Utrecht, The Netherlands; ${ }^{3}$ Department of Surgery, MC Leeuwarden, \\ Leeuwarden, The Netherlands; ${ }^{4}$ Department of Pathology, University Medical Center Utrecht, Utrecht, The Netherlands; \\ ${ }^{5}$ Department of Medical Oncology, University Medical Center Utrecht, Utrecht, The Netherlands
}

\begin{abstract}
Background. Pathological complete response (pCR) following neoadjuvant treatment for locally advanced rectal cancer (LARC) is associated with better survival, less local recurrence, and less distant failure. Furthermore, pCR indicates that the rectum may have been preserved. This meta-analysis gives an overview of available neoadjuvant treatment strategies for LARC and analyzes how these perform in achieving pCR as compared with the standard of care.
\end{abstract}

Methods. Pubmed, Embase, and Cochrane Central bibliographic databases were searched. Randomized controlled trials in which patients received neoadjuvant treatment for MRI-staged nonmetastatic resectable LARC were included. The primary outcome was pCR, defined as ypTONO. A meta-analysis of studies comparing an intervention with standard fluoropyrimidine-based chemoradiation (CRT) was performed.

Results. Of the 17 articles included in the systematic review, 11 were used for the meta-analysis. Addition of oxaliplatin to fluoropyrimidine-based CRT resulted in

Electronic supplementary material The online version of this article (https://doi.org/10.1245/s10434-020-08615-2) contains supplementary material, which is available to authorized users.

(C) The Author(s) 2020

First Received: 21 November 2019;

Published Online: 10 June 2020

S. Hoendervangers, MD

e-mail: s.hoendervangers@umcutrecht.nl significantly more pCR compared with fluoropyrimidinebased CRT only (OR 1.46), but at the expense of more $\geq$ grade 3 toxicity. Other treatment strategies, including consolidation/induction chemotherapy and short-course radiotherapy (SCRT), did not improve pCR rates. None of the included trials reported a benefit in local control or OS. Five-year DFS was significantly worse after SCRT-delay compared with CRT (59\% vs. $75.1 \%$, HR 1.93).

Conclusions. All included trials fail to deliver high-level evidence to show an improvement in pCR compared with standard fluoropyrimidine-based CRT. The addition of oxaliplatin might result in more pCR but at the expense of more toxicity. Furthermore, this benefit does not translate into less local recurrence or improved survival.

The aim of rectal cancer treatment is to improve survival and prevent local recurrence, while limiting treatment-related morbidity and preserving bowel, sexual, and genitourinary function. ${ }^{1,2}$ Consequently, patients with locally advanced rectal cancer (LARC) generally undergo neoadjuvant chemoradiation (CRT) followed by surgery. ${ }^{3,4}$ This combined modality approach decreases recurrence rates and improves survival compared with surgery only. ${ }^{4,5}$ The most frequently used neoadjuvant treatment strategy for LARC is a combination of radiotherapy $(25 \times 2$ Gy or $28 \times 1.8 \mathrm{~Gy})$ and fluoropyrimidine-based chemotherapy [e.g., capecitabine or 5-fluorouracil (5FU)]. Hereby 15-20\% of LARC patients achieve a pathological complete response (pCR) in which no tumor is found in the surgical resection specimen. ${ }^{6-8}$ 
Unfortunately, $30 \%$ of patients who receive this treatment will still die within 5 years due to local or distant recurrence. ${ }^{9}$ However, patients with pCR after neoadjuvant therapy are reported to have better survival, lower local recurrence, and less distant failure rates. ${ }^{10}$ The observation of pCR after surgery has led to a paradigm shift in rectal cancer management, in which organ preservation has become an increasingly important endpoint after neoadjuvant treatment in combination with reduction of local recurrence and survival rates. ${ }^{9}$ Organ-preserving treatment strategies can be considered when a complete response is detected clinically, radiographically, and/or endoscopically before surgery [i.e., clinical complete response (cCR)]. This strategy may protect patients from surgery-associated morbidity and the associated impairment in quality of life. ${ }^{11,12}$ As such, patients with cCR following neoadjuvant treatment are increasingly being offered watch-and-wait regimens or organ-sparing strategies, such as local excision. ${ }^{13,14}$ To further increase the number of eligible patients for such organ preservation strategies, physicians are searching for (new) neoadjuvant treatments with higher organ-sparing potential than the current standard of care.

Previous studies suggested that treatment intensification, i.e., adding chemotherapy or dose-escalated radiotherapy to standard chemoradiation, might enhance rectum preservation and/or improve oncological outcomes. ${ }^{15}$ Theoretically, intensified treatment would further downstage the tumor and any nodal disease prior to surgery and/or target potential micrometastatic disease. ${ }^{4}$ On the contrary, others prefer a short-course (radiation) schedule over long-course chemoradiation, based on its lower toxicity rates, better compliance, and lower cost. ${ }^{16-19}$

The present systematic review and meta-analysis gives an overview of available neoadjuvant treatment strategies for LARC and analyzes how these perform in achieving pCR (as a surrogate endpoint for cCR) compared with the current standard of care in patients with locally advanced rectal cancer based on available evidence from randomized trials.

\section{METHODS}

The present study is registered in the PROSPERO database under number CRD 42017058674.

\section{Search Strategy}

Pubmed, Embase, and Cochrane Central bibliographic databases were searched (last update June 20, 2019) for randomized controlled trials on neoadjuvant treatment for locally advanced rectal cancer, restricted to full text and English language. The search strategy, search syntax, and characteristics of excluded studies are presented in Supplementary Tables 1 and 2 (available online). Crossreferencing was performed.

\section{Eligibility Criteria}

Phase II-III randomized controlled trials (RCTs), conducted after the introduction of total mesorectal excision (TME) surgery in the $1980 \mathrm{~s},{ }^{20}$ in which patients received neoadjuvant treatment for magnetic resonance imaging (MRI)-staged nonmetastatic LARC were included. LARC was defined as stage II-III (cT3-4N0 or T1-4N1-2) rectal cancer. All neoadjuvant treatment modalities that entailed systemic therapy and/or radiotherapy were eligible. Radiotherapy, delivered in either a short course or a long course, was considered suitable, also optionally accompanied by radiation dose escalation. Inclusion was restricted to studies using an interval of at least 4 weeks between end of neoadjuvant therapy and surgery. The primary outcome was pCR, defined as ypT0N0. Studies that did not report ypTN stage were excluded. Secondary outcomes were $\geq$ grade 3 toxicity [according to the Common Terminology Criteria for Adverse Events (CTCAE) version 3.0 or 4.0], surgical outcomes (complication rate and R0 resection rate), local recurrence (LR), disease free survival (DFS), and overall survival (OS). Administration of postoperative systemic therapy was not an exclusion criterion since this could not influence our primary outcome. Study selection was solely based on the primary outcome.

\section{Study Selection}

Identified studies were listed in EndNote (1988-2012 Thomson Reuters). Two authors (S.H. and J.B.) independently screened on title and abstract. Full-text reports were retrieved and examined for eligibility criteria. Studies that only partially fulfilled the eligibility criteria were excluded. Disagreements were resolved by discussion between the two raters. Duplicates were removed, and multiple reports of the same study were linked together. Lastly, the corresponding author of each included study was contacted to obtain additional information or information at individual patient level.

\section{Risk of Bias Assessment}

Risk of bias was assessed by the first author using the Cochrane risk-of-bias tool, ${ }^{21}$ including random sequence generation, allocation concealment, blinding of participants and personnel, blinding of outcome assessment, incomplete outcome data, selective reporting, and other biases. All studies were included in the analyses, irrespective of their risk of bias. 


\section{Data Extraction}

From each included trial, information about trial characteristics (study year/duration and year and country of publication), methodology (phase II or III RCT, number of arms, and sample size), characteristics of study participants [clinical tumor and nodal stage, involvement of the mesorectal fascia (MRF), and distance from the anus in $\mathrm{cm}$ ], characteristics of intervention [agent(s), (radiotherapy) dose, duration, and interval to surgery in weeks], and outcomes $[\mathrm{pCR}$ (ypT0N0) rate, $\geq$ grade 3 toxicity (CTCAE), percentage of patients who received complete dose chemotherapy, percentage of patients that proceeded to surgery, surgical complications, R0 resection rate, and oncological outcomes (LR, DFS, and OS)] was collected. Survival data are reported as 3-year cumulative incidence rates. If available from the report, hazard ratios (HR) are also presented.

\section{Data Analysis}

Four subgroups were created based on neoadjuvant treatment: multiagent chemoradiation $(n=9)$, induction chemotherapy $(n=5), \quad$ consolidation chemotherapy $(n=2)$, and short-course radiotherapy and delayed surgery (SCRT-delay, $n=1$ ). A systematic review of all included studies was performed. A quantitative meta-analysis on the studies that compared an intervention with standard fluoropyrimidine-based chemoradiation $(25-28 \times 1.8-2$ Gy + capecitabine/5FU) was conducted to investigate their effect size. The Mantel-Haenszel random-effects model (REM) was applied, assuming that heterogeneity among studies was not a result of chance alone. Heterogeneity was expressed with $I^{2} .^{22}$ The pooled effect size was calculated from per-protocol data and is expressed as the odds ratio (OR) and its $95 \%$ confidence interval (CI).

All analyses were performed using Review Manager (RevMan), version 5.3 (The Nordic Cochrane Centre, The Cochrane Collaboration, 2014, Copenhagen, Denmark). Results were reported according the Preferred Reporting Items for Systematic Reviews and Meta-analyses (PRISMA) guidelines. ${ }^{23}$

\section{RESULTS}

\section{Eligible Studies}

The literature search obtained 586 records after removal of duplicates, of which 526 records were excluded at title/ abstract screening (Fig. 1). After full-text review, 17 articles met the inclusion and exclusion criteria and were included in the systematic review. Of those, 11 papers were included in the quantitative (meta)analysis. Four studies were excluded from the meta-analysis because these did not include a fluoropyrimidine-based (standard) CRT control arm. Two trials were excluded from quantitative analysis because these were the only ones in their subgroups. ${ }^{19},{ }^{24}$

\section{Risk of Bias}

In general, random sequence generation and allocation concealment were well performed and described (Fig. 2). Participants and personnel were not blinded in most studies. However, this was considered as low risk of bias since the primary outcome pCR was unlikely to be influenced by this. On the contrary, most studies lacked a blinded assessment of $\mathrm{pCR}$, which could have increased the risk of detection (observer) bias.

\section{Characteristics of Included Studies}

Ten phase II and nine phase III trials were conducted between 2001 and 2018 (Table 1). Interval to surgery varied from 4 to 12 weeks after end of neoadjuvant therapy. Detailed patient and tumor characteristics as well as an overview of administered therapy doses are presented in Supplementary Tables 3 and 4 (online accessible). The majority of patients had cT3N + tumors (Supplementary Table 3). MRF involvement was reported in eight studies and varied from 0 to $94.7 \%$. Tumors located $<5 \mathrm{~cm}$ from the anus were present in $4-69.6 \%$ of included patients. The outcomes of included randomized controlled trials stratified by neoadjuvant treatment regimen are presented in Table 2.

\section{Fluoropyrimidine-Based CRT Versus Multiagent CRT}

Nine trials compared fluoropyrimidine-based CRT with multiagent CRT. Six trials (two phase II trials and four phase III trials), including 2502 participants, entered the quantitative analysis. Overall, the pooled OR for pCR after multiagent CRT $(n=1248)$ versus standard CRT $(n=1254)$ was statistically significant at $1.46(95 \% \mathrm{CI}$ $\left.1.18-1.79, I^{2} 0 \%\right)$. Subgroup analysis revealed that the pooled OR resulting from phase II trials was not significant (OR 1.19, 95\% CI $0.56-2.52, I^{2} 34 \%$ ), and the pooled OR from phase III trials remained statistically significant in favor of multiagent CRT (OR 1.50, 95\% CI 1.20-1.87, $I^{2}$ $0 \%$, Fig. 3a).

In five trials, the experimental group received a combination of fluoropyrimidine-based chemotherapy and oxaliplatin. ${ }^{25-29}$ In patients who received fluoropyrimidinebased CRT, $\geq$ grade 3 toxicity occurred in $10.7-40 \%$. In the oxaliplatin CRT group, $\geq$ grade 3 toxicity rates were 


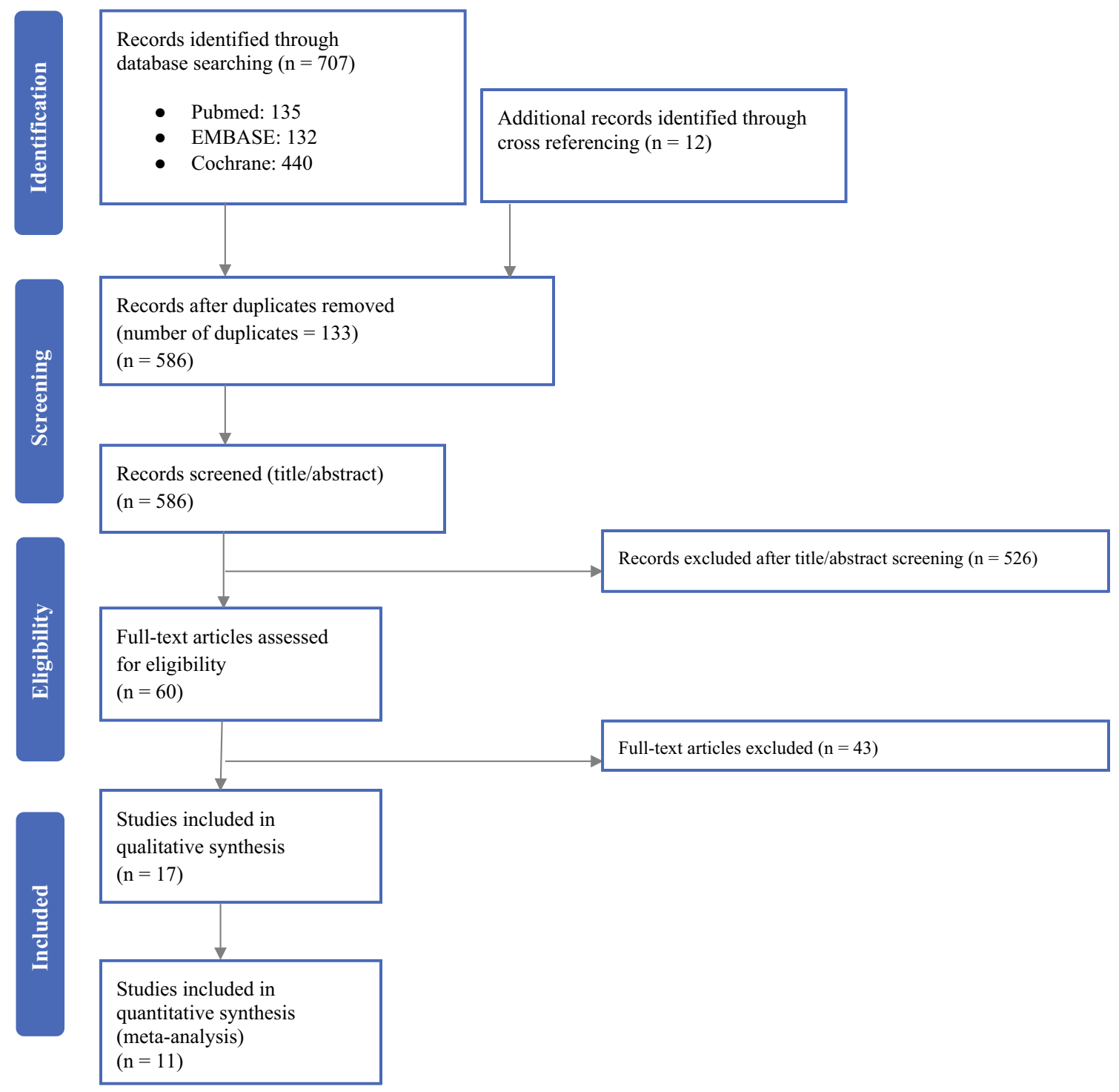

FIG. 1 PRISMA flowchart of included studies. Reasons for exclusion provided as Supplementary Data (available online)

significantly higher (21.4-49.1\%), but this did not affect the number of patients that completed neoadjuvant therapy or the percentage of participants that proceeded to surgery. Neoadjuvant fluoropyrimidine-based CRT resulted in pCR in $13.2-28.3 \%$ of patients. When oxaliplatin was added to this regimen, $\mathrm{pCR}$ rates were $17.4-28.4 \%$. This was statistically significant in two trials. ${ }^{25,29}$ No differences were seen in R0 resections or surgical complications. Two trials compared 5FU-based CRT with multiagent CRT containing irinotecan. ${ }^{30-32}$ One trial described significantly less complete dose administration in the experimental group. ${ }^{30}$ No differences in pCR nor in surgical and survival outcomes were seen. One trial evaluated the effect of targeted therapy (bevacizumab) added to capecitabine-based CRT in 44 patients. $^{24}$ Compared with patients who received capecitabine-based CRT ( $n=46)$, no differences were seen in toxicity or treatment compliance. All but one patient (investigational group) underwent surgery after a median interval of 7.3 weeks. pCR was achieved in $10.9 \%$ of patients in the capecitabine group and $16.3 \%$ of patients in the bevacizumab group. This difference was not statistically significant. Survival data were not available.

For all multiagent comparisons, survival and recurrence data were available from five studies. ${ }^{26,27,29-31}$ No significant differences were reported in LR or OS. Three-year cumulative incidence rates for $\mathrm{LR}$ and $\mathrm{OS}$ in the monotherapy group varied from $4.6-6.1 \%$ to $86.4-88.0 \%$, respectively. For the multiagent group, these rates were $2.9-4.4 \%$ and $88.3-90.3 \%$, respectively. One study reported a significant better 3-year DFS after fluoropyrimidine plus oxaliplatin-based CRT $(71.2 \%$ vs. $75.9 \%$, HR 0.79 , 95\% CI 0.64-0.98, Table 2). ${ }^{29}$ 

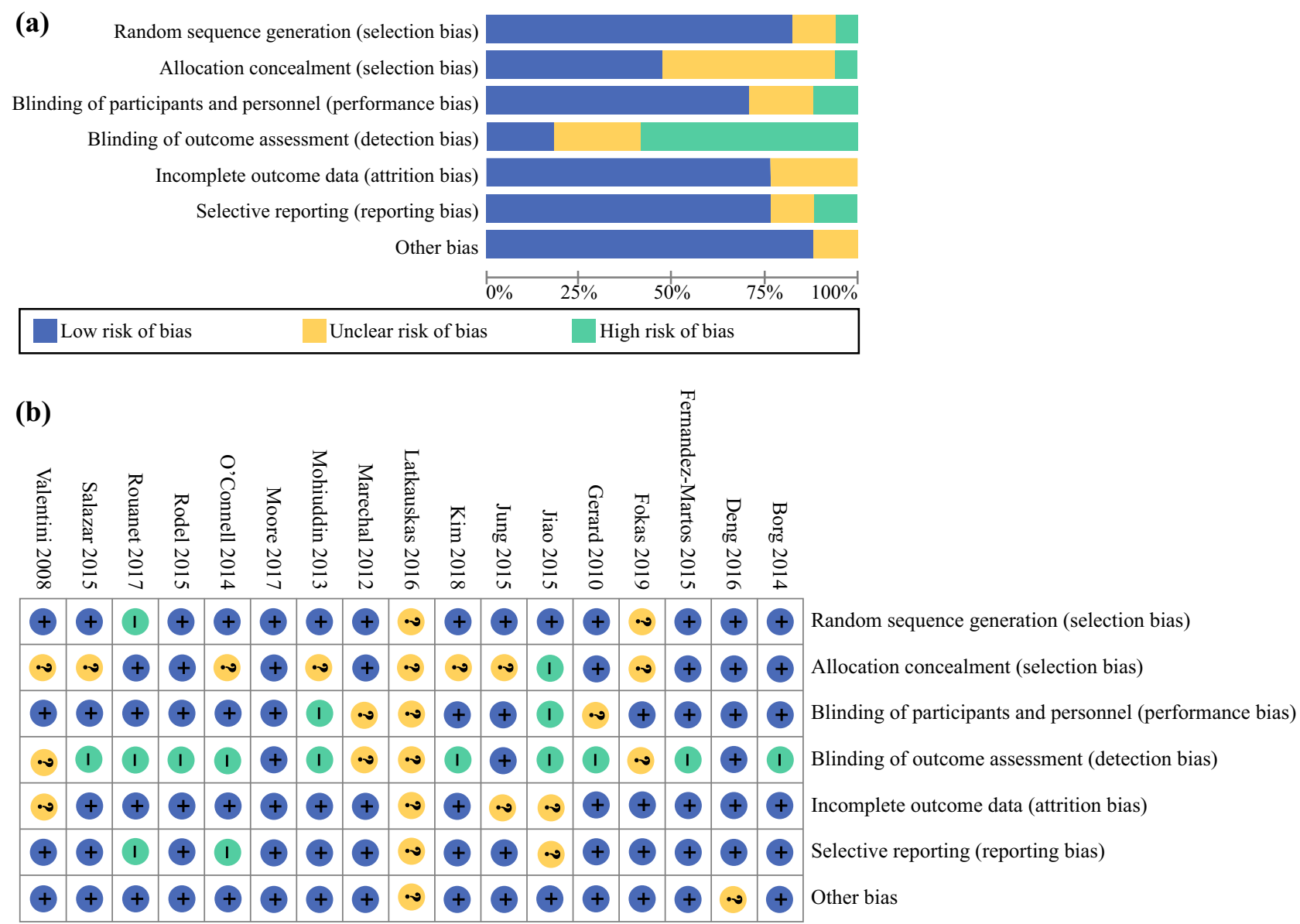

FIG. 2 Review authors' judgements about each risk of bias item presented as percentages across all included studies: a risk of bias graph and b risk of bias summary

\section{Induction Chemotherapy}

Five trials investigated the effect of CRT on pCR when this was preceded by induction chemotherapy. Induction chemotherapy plus CRT was compared with standard CRT in three phase II trials. ${ }^{33-35}$ In these trials, induction therapy consisted of multiagent chemotherapy (i.e., CAPOX or FOLFOX). Toxicity was higher after induction chemotherapy and resulted in significantly lower compliance to CRT in one trial. ${ }^{34,36}$ There were no differences in surgical outcomes or survival. There was no significant difference for pCR after induction chemotherapy $(n=123)$ versus standard CRT $(n=118)$ with a pooled OR of 1.20 (95\% CI 0.62-2.35, $I^{2} 0 \%$, Fig. 3b).

Two trials (GRECCAR-4 and CAO/ARO/AIO-12) in this subgroup were not used for quantitative analysis. The GRECCAR-4 trial randomized patients based on their response to induction FOLFIRINOX ${ }^{37}$ Good responders either received additional capecitabine-based CRT or underwent surgery. Poor responders were randomized to either capecitabine-based CRT or capecitabine-based CRT with dose-escalated radiotherapy (60 Gy). The trial was stopped prematurely due to low accrual rates in the goodresponders arm. In the good-responder arm $(n=20)$, pCR was achieved in 1 of $11(9.1 \%)$ patients after FOLFIRINOX alone and in 11 of 19 (57.9\%) patients after induction chemotherapy with FOLFIRINOX and capecitabine-based CRT. In the poor-responder group $(n=103)$, CRT with dose-escalated radiotherapy resulted in pCR in 9 of $51(17.6 \%)$ patients compared with 7 of $52(13.5 \%)$ patients in the standard-CRT group. This was not a significant difference. The higher radiation dose in the poor responders arm increased R0 resection from 83 to $88 \%$. The CAO/ARO/AIO-12 trial compared CRT and consolidation chemotherapy with CRT and induction therapy. ${ }^{38}$ Acute $\geq$ grade 3 toxicity occurred in $21.8 \%$ and $35.9 \%$ patients after induction chemotherapy alone and CRT after induction chemotherapy, respectively, compared with $27.3 \%$ in participants undergoing CRT before consolidation chemotherapy and $20 \%$ during consolidation therapy. There were no differences in number of R0 resections. pCR was significantly higher in the consolidation group. Longterm survival outcomes were not available. 


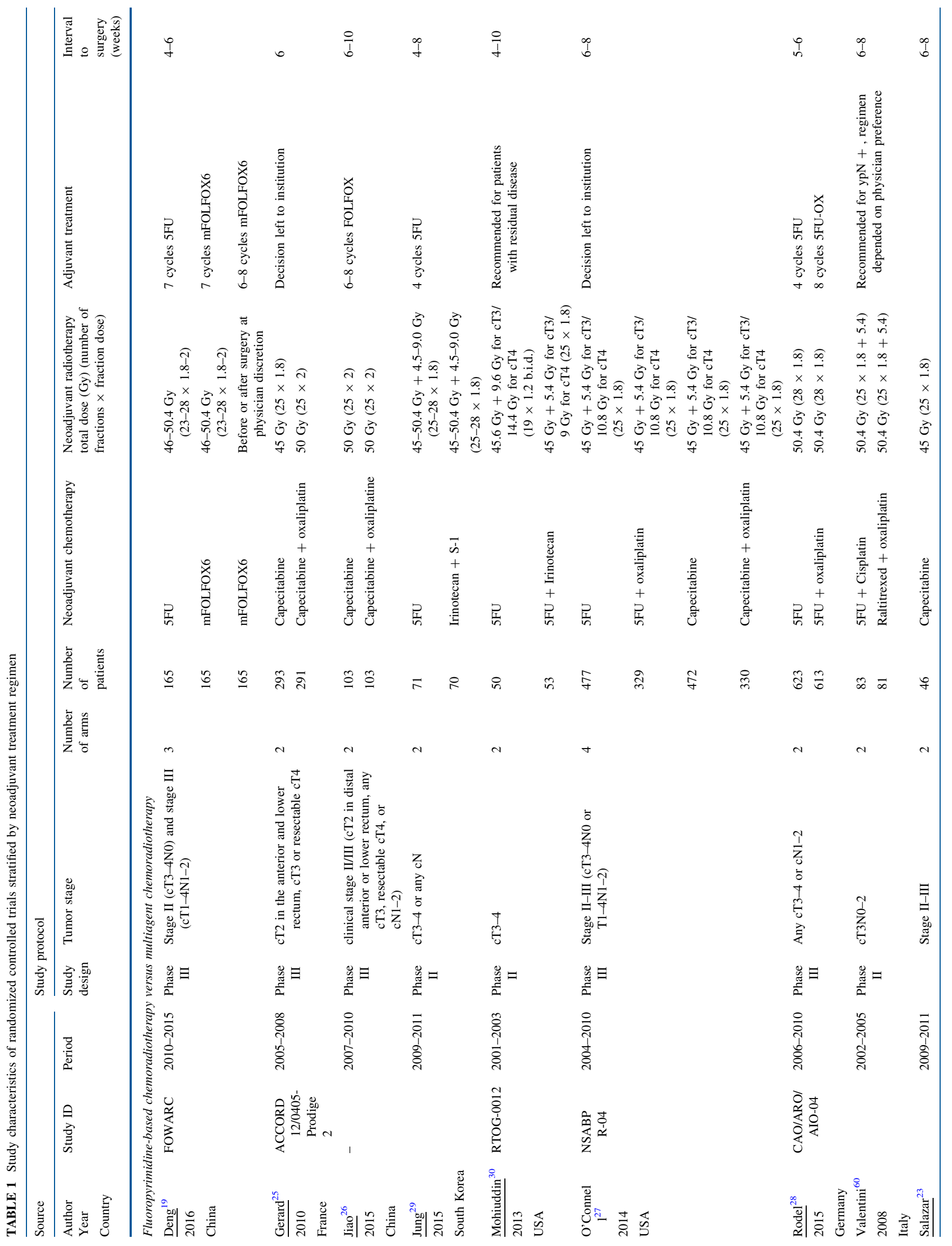




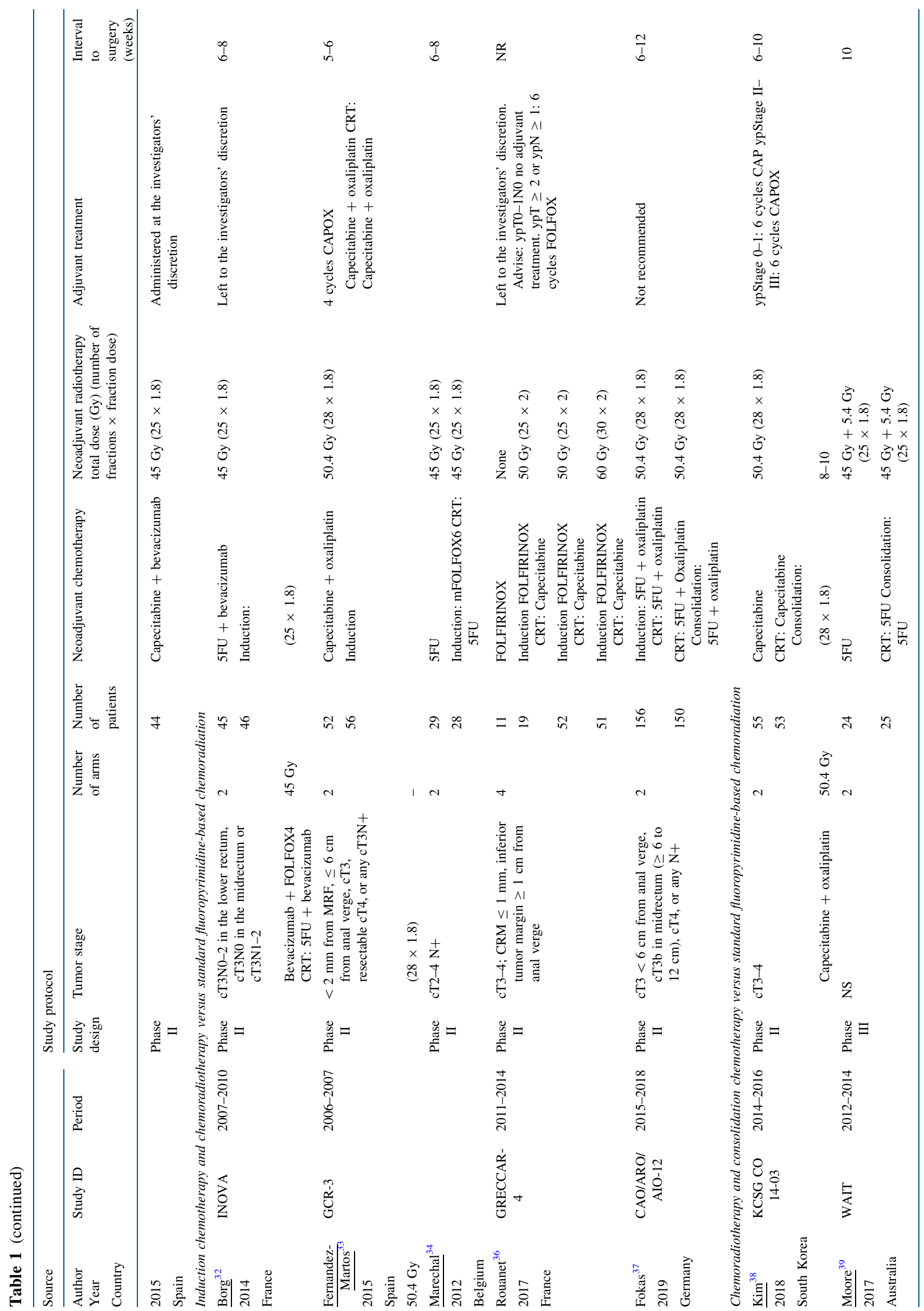


Two RCTs (one phase II and one phase III trial) compared standard CRT with CRT followed by consolidation chemotherapy with either CAPOX or 5FU. ${ }^{39,40}$ Acute $\geq$ grade 3 toxicity was reported in one trial and did not differ between groups. ${ }^{39}$ R0 resections were achieved in 91.7-100\% of patients after standard CRT and 88.6-92\% of patients after CRT with consolidation CAPOX. This was a nonsignificant difference. The quantitative analysis for pCR in standard CRT $(n=76)$ versus CRT with consolidation CAPOX $(n=69)$ resulted in a nonsignificant difference with pooled OR of 1.17 (95\% CI $0.33-4.23, I^{2}$ $54 \%)$. On the subgroup analysis, the phase II trial was in favor of CRT with consolidation therapy (OR 2.58, 95\% CI $0.61-10.99),{ }^{41}$ and the phase III trial was in favor of standard CRT (OR 0.57, 95\% CI 0.14-2.35). ${ }^{40}$ None of the ORs were statistically significant (Fig. 3c). Survival data were not reported.

\section{Short-Course Radiotherapy and Delayed Surgery}

One trial compared SCRT-delay with capecitabinebased CRT, ${ }^{19,42}$ resulting in a nonsignificant different $\mathrm{pCR}$ rate $(4.4 \%$ vs. $11.1 \%$, respectively). There were no differences in radicality or surgical complications. Five-year DFS was significantly worse after SCRT-delay compared with CRT (59\% vs. $75.1 \%$, HR 1.93, Table 2).

\section{DISCUSSION}

This systematic review evaluates whether pCR rates are higher following alternative neoadjuvant treatment strategies as compared with standard neoadjuvant fluoropyrimidine-based chemoradiation. All included trials fail to deliver high-level evidence to show an improvement in pathological outcomes or survival compared with standard fluoropyrimidine-based CRT. The addition of oxaliplatin to fluoropyrimidine-based CRT might result in significantly more $\mathrm{pCR}$, but at the expense of more $\geq$ grade 3 toxicity. Furthermore, this benefit does not translate into lower rates of local recurrence or improved overall survival. Other neoadjuvant treatment strategies, including consolidation/induction chemotherapy and short-course radiotherapy with delayed surgery, were not associated with improved pCR rates. None of the included trials reported benefit in local recurrence or overall survival.

pCR following neoadjuvant therapy has been associated with improved survival ${ }^{7}$ and may reflect the organ-sparing potential of a treatment protocol. To increase clinical response rates after neoadjuvant treatment and herewith enable rectum preservation, different intensification strategies have been investigated in phase I-II trials, e.g., 


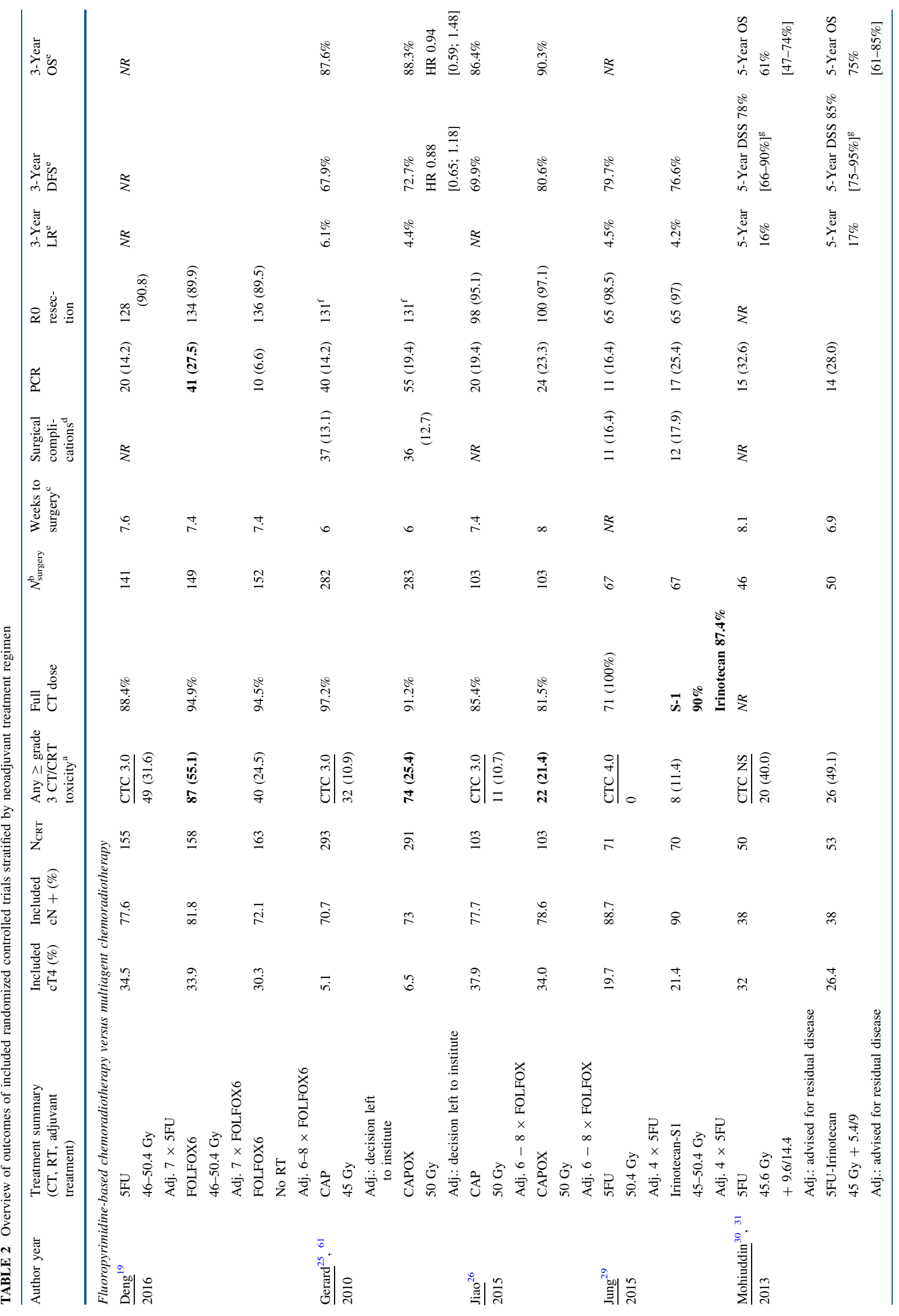




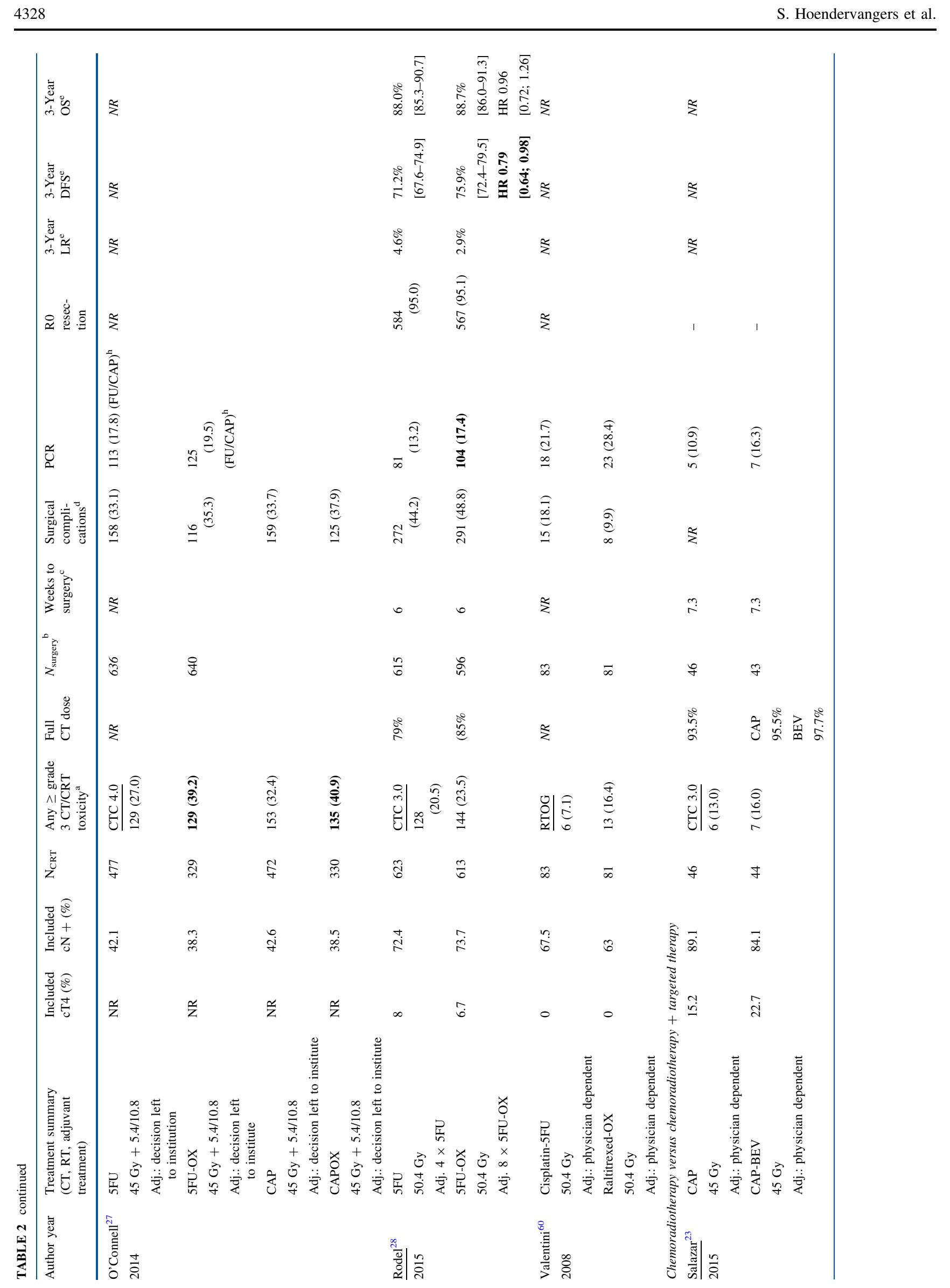




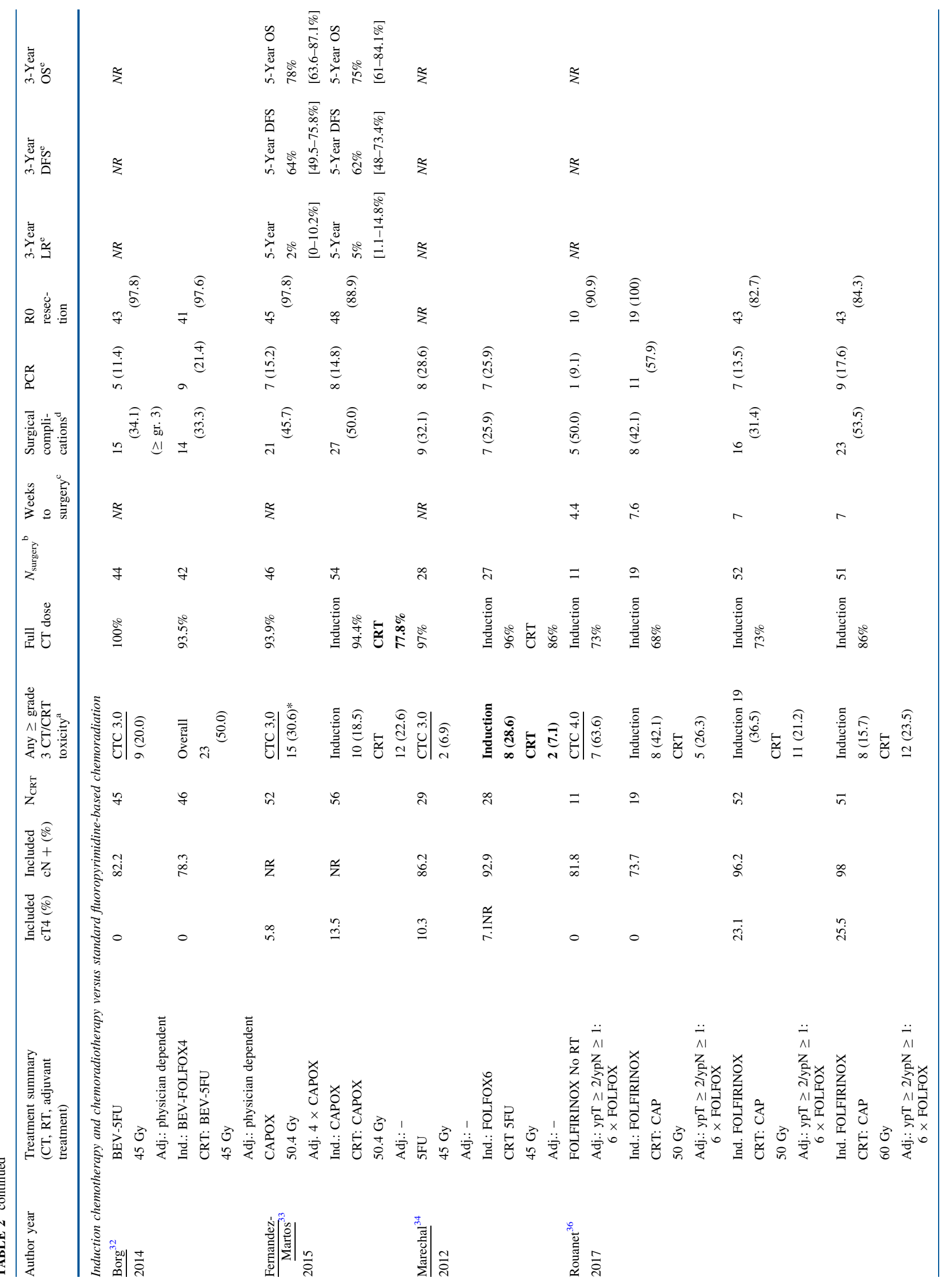




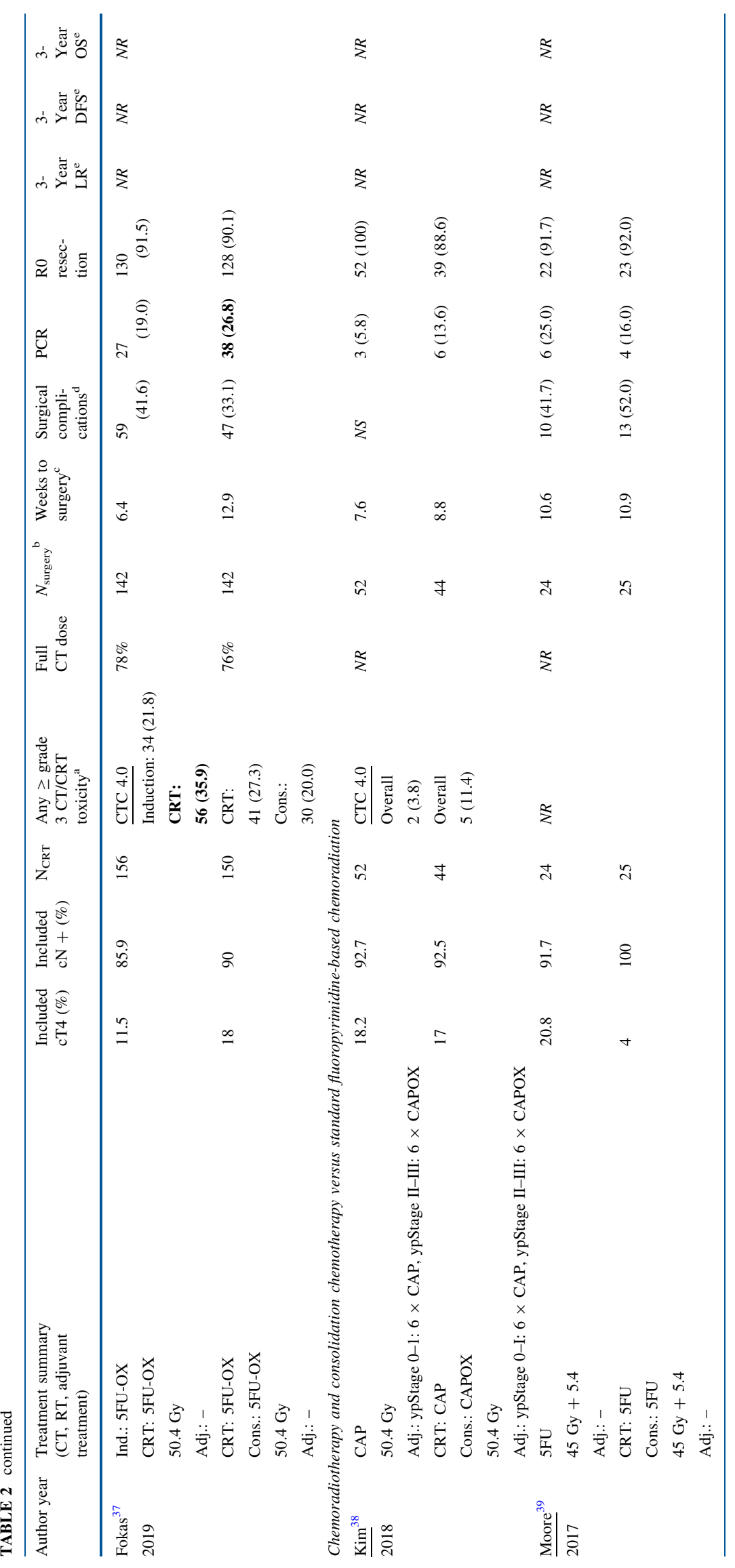


pCR Following Neoadjuvant Therapy for LARC

4331

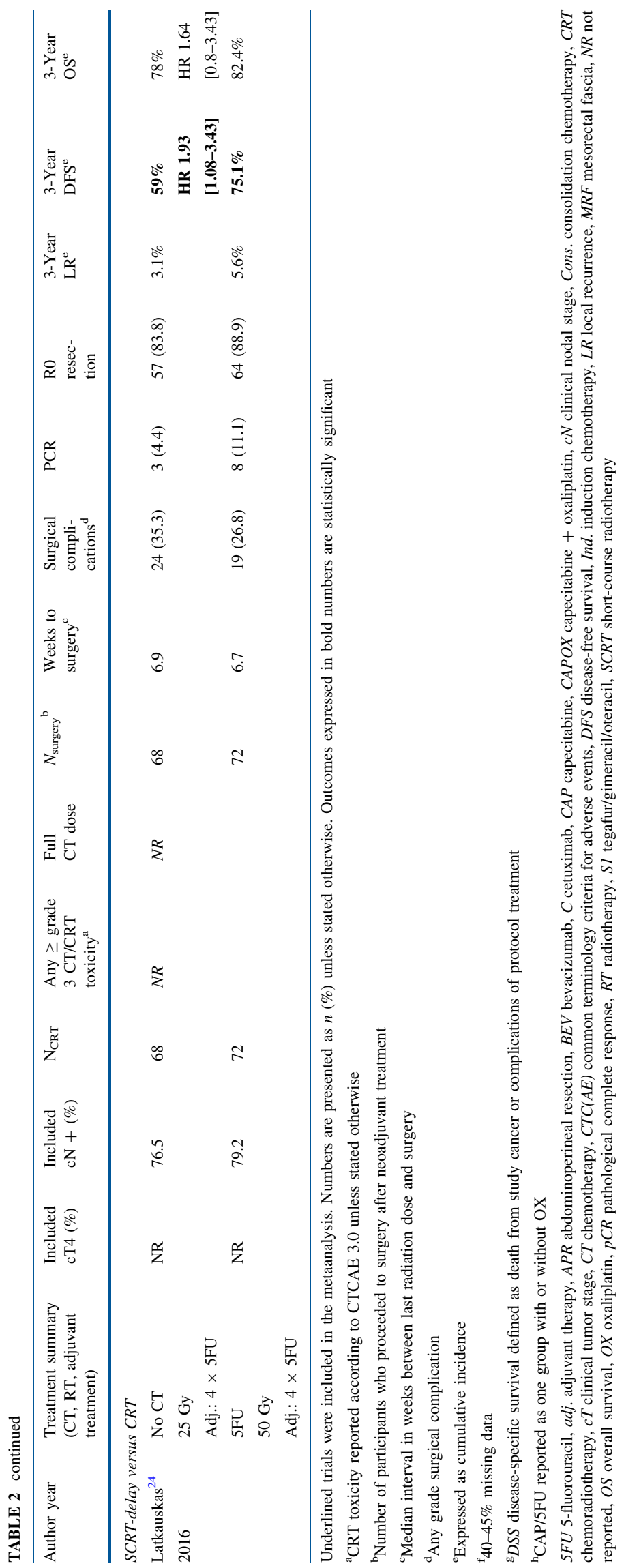


(a) Multi-agent chemoradiation vs. standard fluropyimidine-based chemoradiation, analyzed per type

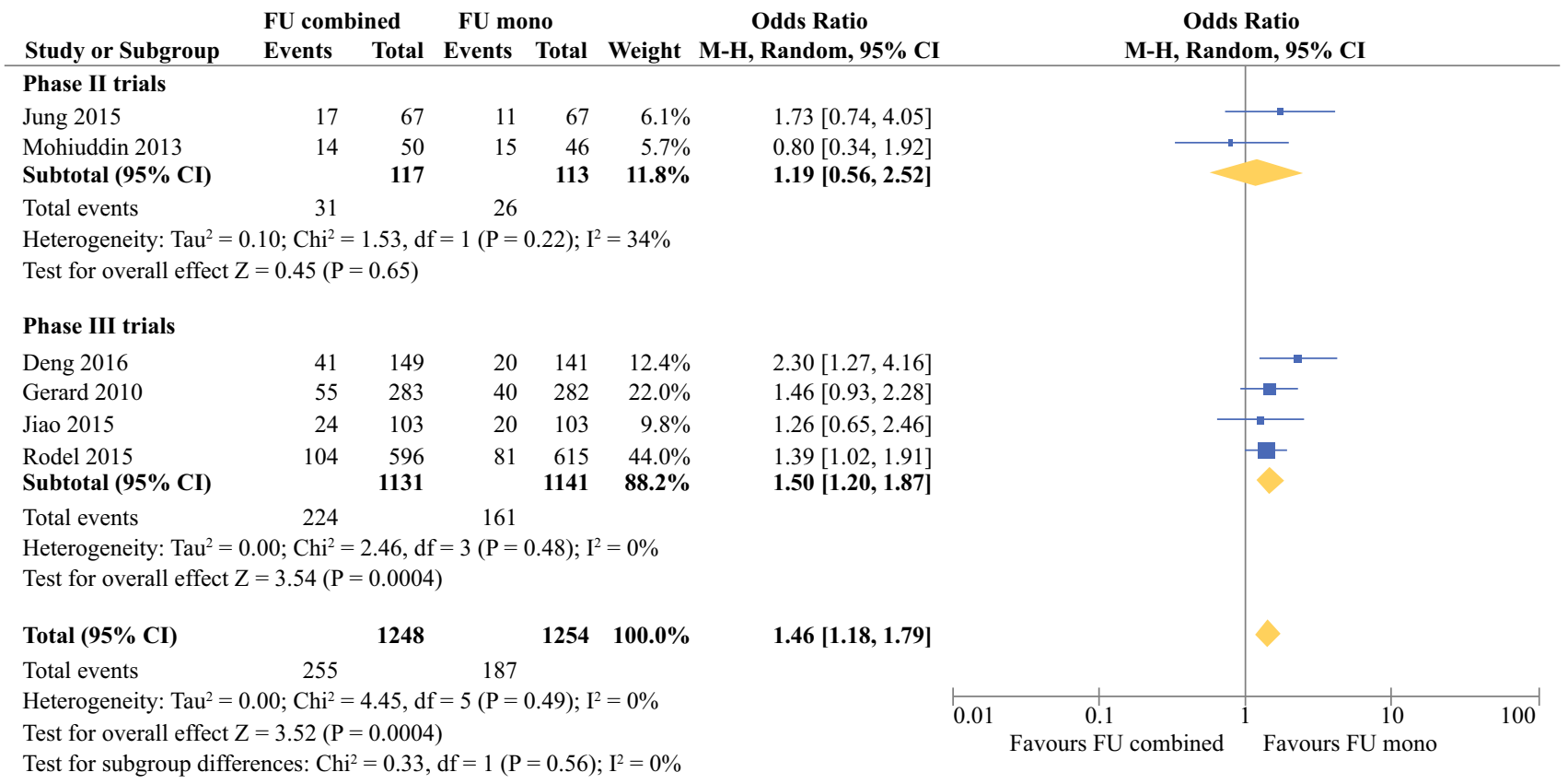

(b) Induction chemotherapy and chemoradiotherapy vs. standard fluropyimidine-based chemoradiation

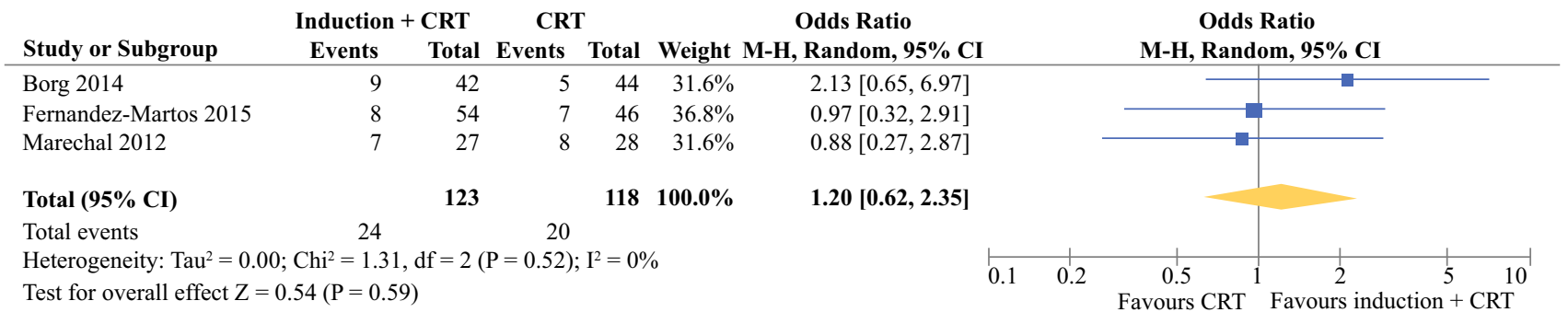

(c) Chemoradiotherapy and consolidation chemotherapy vs. standard fluropyimidine-based chemoradiation, analyzed per trial type

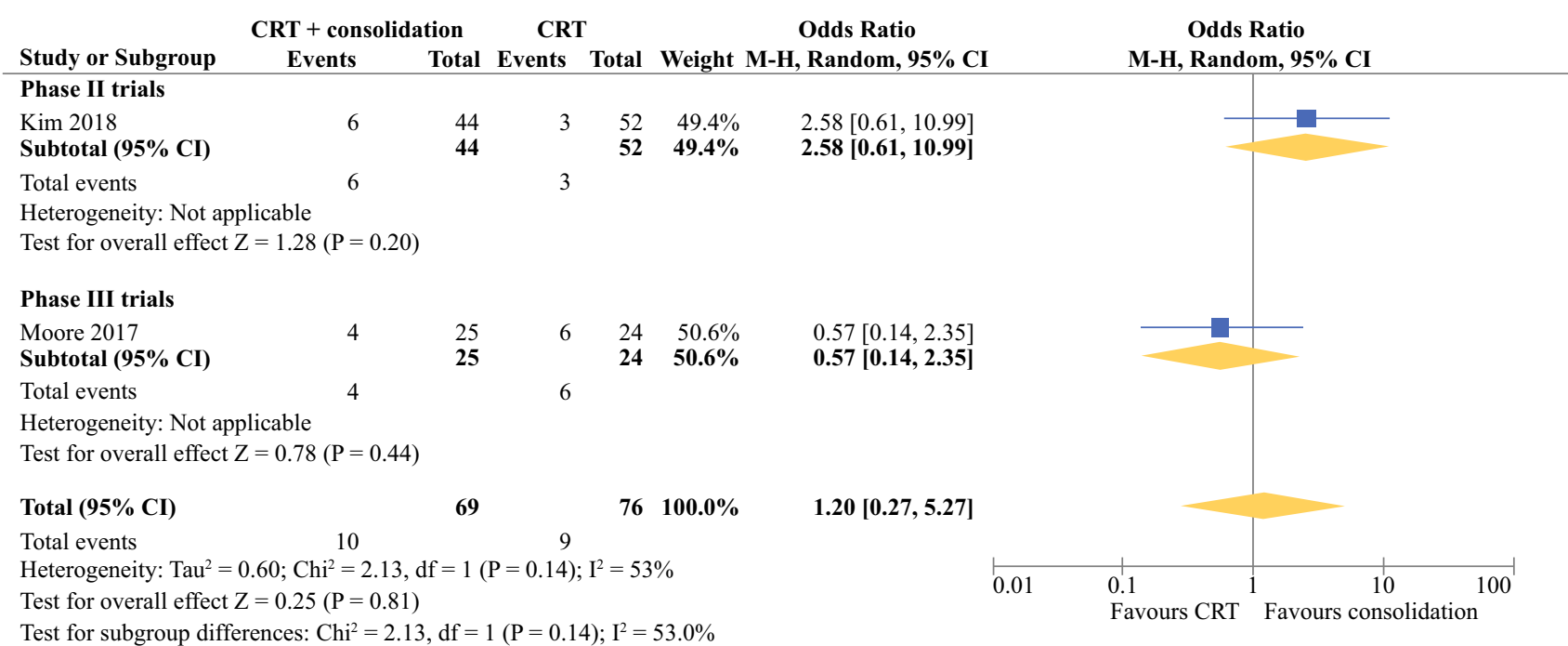

FIG. 3 Pooled OR of pCR rates following multiagent chemoradiation, consolidation chemotherapy, and induction chemotherapy compared with standard fluoropyrimidine-based CRT 
multiagent CRT, targeted therapy, radiotherapy doseescalation, or additional chemotherapy before or after CRT [total neoadjuvant treatment (TNT)]. On multivariable metaregression, the addition of a second concurrent chemotherapy agent was not associated with improved pCR rates. ${ }^{43}$ In accordance with our findings, previous meta-analyses showed that the addition of oxaliplatin to preoperative chemoradiotherapy improves $\mathrm{pCR}$ rate, decreases LR rate, and improves DFS, but significantly worsens toxicity. ${ }^{44,45}$ Also, no significant difference was found in the $\mathrm{R} 0$ resection rate, sphincter preservation rate, permanent stoma rate, postoperative complication, mortality, or overall survival. ${ }^{45}$ Dose-escalated radiotherapy could be associated with higher pCR rates. ${ }^{43,46}$ However, this has not yet been confirmed by a randomized controlled trial and could therefore not be further investigated in the present study. ${ }^{6}$ TNT might manage micrometastases, increase tumor regression that enhances $\mathrm{R} 0$ resection rates, and increase probabilities for organ preservation. ${ }^{38} \mathrm{~A}$ recent meta-analysis showed that patients who received TNT followed by surgery more often achieved pCR (OR $1.39,1.08-1.81)$ and better DFS (HR 0.75, 0.52-1.07) and OS (HR $0.73(0.59-0.9)$ than those who received CRT only. However, this analysis was largely based on nonrandomized comparative studies, and in subgroup analyses (prospective and retrospective series), there were no statistically significant differences between TNT and CRT arms. ${ }^{15}$ Several trials are still ongoing, ${ }^{47,48}$ but to date, the superiority of TNT over standard CRT remains inconclusive.

Targeted therapy is the latest development in rectal cancer management. Translational research has led to better understanding of molecular pathways and increased the interest in targeted therapy; For example, cancer cells can express epidermal growth factor receptor (EGFR), which stimulates cell proliferation, as well as vascular endothelial growth factor receptor (VEGFR), enabling vessel formation for growth, ${ }^{49,50}$ and EGFR signaling might promote resistance to radiotherapy. Retrospective analyses demonstrated worse DFS and lower pCR rates in patients with rectal tumors expressing EGFR, and elevated VEGF expression in tumors has been associated with inferior survival. ${ }^{49}$ The addition of cetuximab, a monoclonal antibody that can sensitize cells with overexpression of EGFR to radiotherapy, ${ }^{49}$ has been shown not to affect the $\mathrm{pCR}$ rate but to significantly improve OS. ${ }^{51}$ Bevacizumab, an anti-VEGF antibody reducing tumor vascular density, ${ }^{49,} 50$ did not improve pCR rates. ${ }^{24}$ However, these translational results are still preliminary, and clinical trials are needed.

In specific patient populations (elderly or frail) or in some countries, SCRT-delay is preferred over CRT because of its lower costs, better compliance, and less demanding nature. ${ }^{52}$ However, the use of SCRT remains elusive outside of Europe. ${ }^{9}$ Unsurprisingly, pCR rates are lower with this regimen based on its lower biological effective radiation dose compared with long-course chemoradiation. The largest randomized trial that investigated the effect of SCRT-delay was the Swedish Stockholm III trial. ${ }^{53}$ pCR was found in $10.4 \%$ of patients after SCRT-delay, and the risk of postoperative complications was significantly lower after SCRT-delay compared with SCRT and immediate surgery. ${ }^{18,54}$ However, this trial could not be included in the present study due to the lack of baseline tumor characteristics. Additionally, a combination of (induction/consolidation) chemotherapy and SCRT-delay could increase pCR rates and improve survival. ${ }^{42,55,56}$ The results of a large RCT on this topic are still awaited. ${ }^{56}$ Therefore, at this moment, SCRT-delay only seems appropriate for frail LARC patients who are unfit to undergo CRT.

This is the first systematic review to provide an overview of the most widely used and available neoadjuvant treatment modalities investigated in a randomized trial. The evaluation of pathological outcomes in relation to toxicity and surgical and survival data provides more insight in the overall effect of these regimens. Nonetheless, this meta-analysis also encountered several limitations. First, only RCTs were included, whereas a lot of new interventions are trialed in prospective single-arm phase II trials. However, these trials are prone to selection bias as well as optimism in the intervention effect and often fail to demonstrate superiority in subsequent phase III trials. ${ }^{43,57}$, 58 Nonetheless, randomized phase II trials may also overestimate the treatment effect. ${ }^{59}$ We showed these differences between phase II and phase III trials in the analyses for multiagent CRT and for CRT plus consolidation chemotherapy. In addition, the RCT-limited analysis might represent a relatively well-conditioned study population, ${ }^{60}$ resulting in an underestimation of compliance and toxicity rates. Second, the generalizability might be limited due to strict MRI criteria and pCR definitions. Although MRI is considered to be the most optimal staging method, ${ }^{2,61}$ this may not be as widely available and easy accessible in all countries. In addition, the primary outcome was restricted to ypT0N0 because the interobserver agreement of other methods for tumor regression grading is low. ${ }^{62}$ The tumor regression grade (TRG) definition of pCR varies among approaches, and the application of a TRG is not recommended in the present TNM classification. ${ }^{62,63}$ Moreover, subgroups were small, and secondary outcomes could not be extracted from all included trials, which might reduce power. Third, despite strict inclusion criteria and the use of a random-effects model, uncorrected heterogeneity in study protocols might still influence the pooled effect estimates. ${ }^{64}$ This is for instance reflected in the different 
intervals between the end of neoadjuvant treatment and surgery. A prolonged interval may increase pCR rates and recurrence-free survival without compromising surgical morbidity. ${ }^{65,66}$ As such, higher pCR rates after consolidation therapy compared with induction therapy may be the result of an increased interval between surgery and CRT rather than the therapy itself. And lastly, only those treatments compared with a similar baseline, namely standard fluoropyrimidine-based CRT, could be used in a formal meta-analysis. The opportunity to perform an extended network meta-analysis was explored but was not reliable due to the large heterogeneity in study design and the small amount of available RCTs.

The currently available data show that there is a wide variety of neoadjuvant treatment strategies available but no high-level evidence to show an improvement in pathological outcomes and survival compared with standard of care in terms of pCR achievement and organ-sparing potential. This is probably caused by the large number of confounding factors resulting from differences in diagnosis and treatment but, more importantly, also from differences in patient and tumor characteristics. In the era of personalized treatment, more high-level evidence on tumor characteristics, (pre)treatment response prediction, longterm quality of life, and oncological outcomes after different treatment modalities is needed to support optimal and individualized rectal cancer management. This requires new, efficient, and innovative research infrastructures, such as large prospective cohorts in which trials can be conducted according to the "Trials within Cohorts" (TwiCs) design. ${ }^{67,68}$ This enables investigation of novel prognostic and predictive factors in large populations as well as in small subgroups of patients and simultaneously provides the platform to conduct (partly) overlapping randomized trials with robust and validated analysis methods that provide clinically relevant answers that can be directly translated into changes for routine care. ${ }^{69}$

ACKNOWLEDGMENT The authors thank all approached authors that cooperated in sharing their data for this analysis.

\section{DISCLOSURES None.}

OPEN ACCESS This article is licensed under a Creative Commons Attribution 4.0 International License, which permits use, sharing, adaptation, distribution and reproduction in any medium or format, as long as you give appropriate credit to the original author(s) and the source, provide a link to the Creative Commons licence, and indicate if changes were made. The images or other third party material in this article are included in the article's Creative Commons licence, unless indicated otherwise in a credit line to the material. If material is not included in the article's Creative Commons licence and your intended use is not permitted by statutory regulation or exceeds the permitted use, you will need to obtain permission directly from the copyright holder. To view a copy of this licence, visit http://creativecommons. org/licenses/by/4.0/.

\section{REFERENCES}

1. Glynne-Jones R, Wyrwicz L, Tiret E, et al. Rectal cancer: ESMO Clinical Practice Guidelines for diagnosis, treatment and followup. Ann Oncol. 2017;28(suppl_4):iv22-40.

2. Lutz MP, Zalcberg JR, Glynne-Jones R, et al. Second St. Gallen European Organisation for research and treatment of cancer gastrointestinal cancer conference: consensus recommendations on controversial issues in the primary treatment of rectal cancer. Eur J Cancer. 2016;63:11-24.

3. Bosset JF, Collette L, Calais G, et al. Chemotherapy with preoperative radiotherapy in rectal cancer. New Eng J Med. 2006;355(11):1114-23.

4. Nussbaum N, Altomare I. The neoadjuvant treatment of rectal cancer: a review. Curr Oncol Rep. 2015;17(3):434.

5. Zhao F, Wang J, Yu H, et al. Neoadjuvant radiotherapy improves overall survival for T3/4N + M0 rectal cancer patients: a population-based study of 20300 patients. Rad Oncol. 2020;15(1):49.

6. Burbach JP, Verkooijen HM, Intven M, et al. RandomizEd controlled trial for pre-operAtive dose-escaLation BOOST in locally advanced rectal cancer (RECTAL BOOST study): study protocol for a randomized controlled trial. Trials. 2015;16:58.

7. Maas M, Nelemans PJ, Valentini V, et al. Long-term outcome in patients with a pathological complete response after chemoradiation for rectal cancer: a pooled analysis of individual patient data. Lancet Oncol. 2010;11(9):835-44.

8. Sanghera P, Wong DW, McConkey CC, Geh JI, Hartley A. Chemoradiotherapy for rectal cancer: an updated analysis of factors affecting pathological response. Clin Oncol. 2008;20(2):176-83.

9. Aklilu M, Eng C. The current landscape of locally advanced rectal cancer. Nat Rev Clin Oncol. 2011;8(11):649-59.

10. Martin ST, Heneghan HM, Winter DC. Systematic review and meta-analysis of outcomes following pathological complete response to neoadjuvant chemoradiotherapy for rectal cancer. $\mathrm{Br}$ J Surg. 2012;99(7):918-28.

11. Garcia-Aguilar J, Glynne-Jones R, Schrag D. Multimodal rectal cancer treatment: in some cases, less may be more. Am Soc Clin Oncol. 2016;35:92-102.

12. Alves A, Panis Y, Mathieu P, et al. Mortality and morbidity after surgery of mid and low rectal cancer. Results of a French prospective multicentric study. Gastroenterol Clin Biol. 2005;29(5):509-14.

13. Dossa F, Chesney TR, Acuna SA, Baxter NN. A watch-and-wait approach for locally advanced rectal cancer after a clinical complete response following neoadjuvant chemoradiation: a systematic review and meta-analysis. Lancet Gastroenterol Hepatol. 2017;2(7):501-13.

14. Habr-Gama A, Perez RO, Nadalin W, et al. Operative versus nonoperative treatment for stage 0 distal rectal cancer following chemoradiation therapy: long-term results. Ann Surg. 2004;240(4):711-17; discussion 717-18.

15. Petrelli F, Trevisan F, Cabiddu M, et al. Total neoadjuvant therapy in rectal cancer: a systematic review and metaanalysisof treatment outcomes. Ann Surg. 2020;271(3):440-8.

16. Bujko K, Nowacki MP, Nasierowska-Guttmejer A, Michalski W, Bebenek M, Kryj M. Long-term results of a randomized trial comparing preoperative short-course radiotherapy with preoperative conventionally fractionated chemoradiation for rectal cancer. Br J Surg. 2006;93(10):1215-23. 
17. Bujko K, Pietrzak L, Partycki M, et al. The feasibility of shortcourse radiotherapy in a watch-and-wait policy for rectal cancer. Acta Oncol. 2017;56(9):1152-4.

18. Erlandsson J, Holm T, Pettersson D, et al. Optimal fractionation of preoperative radiotherapy and timing to surgery for rectal cancer (Stockholm III): a multicentre, randomised, non-blinded, phase 3, non-inferiority trial. Lancet Oncol. 2017;18(3):336-46.

19. Latkauskas T, Pauzas H, Kairevice L, et al. Preoperative conventional chemoradiotherapy versus short-course radiotherapy with delayed surgery for rectal cancer: results of a randomized controlled trial. BMC Cancer. 2016;16(1):927.

20. Heald RJ, Moran BJ, Ryall RD, Sexton R, MacFarlane JK. Rectal cancer: the Basingstoke experience of total mesorectal excision, 1978-1997. Arch Surg. 1998;133(8):894-9.

21. Higgins JP, Altman DG, Gotzsche PC, et al. The Cochrane Collaboration's tool for assessing risk of bias in randomised trials. BMJ. 2011;343:d5928.

22. Higgins JP, Thompson SG. Quantifying heterogeneity in a metaanalysis. Stat Med. 2002;21(11):1539-58.

23. Moher D, Liberati A, Tetzlaff J, Altman DG, Group P. Preferred reporting items for systematic reviews and meta-analyses: the PRISMA statement. BMJ. 2009;339:b2535.

24. Salazar R, Capdevila J, Laquente B, et al. A randomized phase II study of capecitabine-based chemoradiation with or without bevacizumab in resectable locally advanced rectal cancer: clinical and biological features. BMC Cancer. 2015;15:60.

25. Deng Y, Chi P, Lan P, et al. Modified FOLFOX6 with or without radiation versus fluorouracil and leucovorin with radiation in neoadjuvant treatment of locally advanced rectal cancer: initial results of the chinese FOWARC multicenter, open-label, randomized three-arm phase III trial. $J$ Clin Oncol. 2016;34(27):3300-7.

26. Gerard JP, Azria D, Gourgou-Bourgade S, et al. Comparison of two neoadjuvant chemoradiotherapy regimens for locally advanced rectal cancer: results of the phase III trial ACCORD 12/0405-Prodige 2. J Clin Oncol. 2010;28(10):1638-44.

27. Jiao D, Zhang R, Gong Z, et al. Fluorouracil-based preoperative chemoradiotherapy with or without oxaliplatin for stage ii/iii rectal cancer: a 3-year follow-up study. Chin J Cancer Res. 2015;27(6):588-96.

28. O'Connell MJ, Colangelo LH, Beart RW, et al. Capecitabine and oxaliplatin in the preoperative multimodality treatment of rectal cancer: surgical end points from National Surgical Adjuvant Breast and Bowel Project trial R-04. J Clin Oncol. 2014;32(18):1927-34.

29. Rodel C, Graeven U, Fietkau R, et al. Oxaliplatin added to fluorouracil-based preoperative chemoradiotherapy and postoperative chemotherapy of locally advanced rectal cancer (the German CAO/ARO/AIO-04 study): final results of the multicentre, open-label, randomised, phase 3 trial. Lancet Oncol. 2015;16(8):979-89.

30. Jung M, Shin SJ, Koom WS, et al. A Randomized Phase 2 Study of neoadjuvant chemoradiaton therapy with 5-fluorouracil/leucovorin or irinotecan/S-1 in patients with locally advanced rectal cancer. Int J Radiat Oncol Biol Phys. 2015;93(5):1015-22.

31. Mohiuddin M, Paulus R, Mitchell E, et al. Neoadjuvant chemoradiation for distal rectal cancer: 5-year updated results of a randomized phase 2 study of neoadjuvant combined modality chemoradiation for distal rectal cancer. Int J Radiat Oncol Biol Phys. 2013;86(3):523-28.

32. Mohiuddin M, Winter K, Mitchell E, et al. Randomized phase II study of neoadjuvant combined-modality chemoradiation for distal rectal cancer: radiation therapy oncology group trial 0012. $J$ Clin Ocol. 2006;24(4):650-5.

33. Borg C, André T, Mantion G, et al. Pathological response and safety of two neoadjuvant strategies with bevacizumab in MRI- defined locally advanced $\mathrm{T} 3$ resectable rectal cancer: a randomized, noncomparative phase II study. Ann Oncol. 2014;25(11):2205-10.

34. Fernandez-Martos C, Garcia-Albeniz X, Pericay C, et al. Chemoradiation, surgery and adjuvant chemotherapy versus induction chemotherapy followed by chemoradiation and surgery: long-term results of the Spanish GCR-3 phase II randomized trial†. Ann Oncol. 2015;26(8):1722-8.

35. Marechal R, Vos B, Polus M, et al. Short course chemotherapy followed by concomitant chemoradiotherapy and surgery in locally advanced rectal cancer: a randomized multicentric phase II study. Ann Oncol. 2012;23(6):1525-30.

36. Fernández-Martos C, Pericay C, Aparicio J, et al. Phase II, randomized study of concomitant chemoradiotherapy followed by surgery and adjuvant capecitabine plus oxaliplatin (CAPOX) compared with induction CAPOX followed by concomitant chemoradiotherapy and surgery in magnetic resonance imagingdefined, locally advanced rectal cancer: grupo cancer de recto 3 study. J Clin Oncol. 2010;28(5):859-e.

37. Rouanet P, Rullier E, Lelong B, et al. Tailored treatment strategy for locally advanced rectal carcinoma based on the tumor response to induction chemotherapy: preliminary results of the French phase II multicenter GRECCAR4 trial. Dis Colon Rectum. 2017;60(7):653-63.

38. Fokas E, Allgauer M, Polat B, et al. Randomized phase II trial of chemoradiotherapy plus induction or consolidation chemotherapy as total neoadjuvant therapy for locally advanced rectal cancer: CAO/ARO/AIO-12. J Clin Oncol. 2019:JCO1900308.

39. Kim SY, Joo J, Kim TW, et al. A randomized phase 2 trial of consolidation chemotherapy after preoperative chemoradiation therapy versus chemoradiation therapy alone for locally advanced rectal cancer: KCSG CO 14-03. Int J Radiat Oncol Biol Phys. 2018;101(4):889-99.

40. Moore J, Price T, Carruthers S, et al. Prospective randomized trial of neoadjuvant chemotherapy during the 'wait period' following preoperative chemoradiotherapy for rectal cancer: results of the WAIT trial. Colorectal Dis. 2017;19(11):973-79.

41. Kim SY, Joo J, Kim TW, et al. A randomized phase 2 trial of consolidation chemotherapy after preoperative chemoradiation therapy versus chemoradiation therapy alone for locally advanced rectal cancer: KCSG CO 14-03. Int $J$ Radiat Oncol. 2018;101(4):889-99.

42. Bujko K, Wyrwicz L, Rutkowski A, et al. Long-course oxaliplatin-based preoperative chemoradiation versus $5 \times 5$ Gy and consolidation chemotherapy for cT4 or fixed cT3 rectal cancer: results of a randomized phase III study. Ann Oncol. 2016;27(5):834-42.

43. Teo MTW, McParland L, Appelt AL, Sebag-Montefiore D. Phase 2 neoadjuvant treatment intensification trials in rectal cancer: a systematic review. Int $J$ Radiat Oncol Biol Phys. 2018;100(1):146-58.

44. Huttner FJ, Probst P, Kalkum E, et al. Addition of platinum derivatives to fluoropyrimidine-based neoadjuvant chemoradiotherapy for stage II/III rectal cancer: systematic review and metaanalysis. J Natl Cancer Inst. 2019;111(9):887-902.

45. Zheng J, Feng X, Hu W, Wang J, Li Y. Systematic review and meta-analysis of preoperative chemoradiotherapy with or without oxaliplatin in locally advanced rectal cancer. Medicine. 2017;96(13):e6487.

46. Burbach JP, den Harder AM, Intven M, van Vulpen M, Verkooijen HM, Reerink O. Impact of radiotherapy boost on pathological complete response in patients with locally advanced rectal cancer: a systematic review and meta-analysis. Radiother Oncol. 2014;113(1):1-9.

47. Schrag D, Weiser M, Saltz L, et al. Challenges and solutions in the design and execution of the PROSPECT Phase II/III 
neoadjuvant rectal cancer trial (NCCTG N1048/Alliance). Clin Trials. 2019;16(2):165-75.

48. Smith JJ, Chow OS, Gollub MJ, et al. Organ preservation in rectal adenocarcinoma: a phase II randomized controlled trial evaluating 3-year disease-free survival in patients with locally advanced rectal cancer treated with chemoradiation plus induction or consolidation chemotherapy, and total mesorectal excision or nonoperative management. BMC Cancer. 2015;15:767.

49. Wadlow RC, Ryan DP. The role of targeted agents in preoperative chemoradiation for rectal cancer. Cancer. 2010;116(15):3537-48.

50. Jain RK. Normalization of tumor vasculature: an emerging concept in antiangiogenic therapy. Science. 2005;307(5706):58-62.

51. Dewdney A, Cunningham D, Tabernero J, et al. Multicenter randomized phase II clinical trial comparing neoadjuvant oxaliplatin, capecitabine, and preoperative radiotherapy with or without cetuximab followed by total mesorectal excision in patients with high-risk rectal cancer (EXPERT-C). J Clin Oncol. 2012;30(14):1620-7.

52. Bujko K, Bujko M. Point: short-course radiation therapy is preferable in the neoadjuvant treatment of rectal cancer. Semin Radiat Oncol. 2011;21(3):220-7.

53. Pettersson D, Lorinc E, Holm T, et al. Tumour regression in the randomized Stockholm III trial of radiotherapy regimens for rectal cancer. Br J Surg. 2015;102(8):972-8; discussion 978.

54. Erlandsson J, Lorinc E, Ahlberg M, et al. Tumour regression after radiotherapy for rectal cancer-results from the randomised Stockholm III trial. Radiother Oncol. 2019;135:178-86.

55. Gollins S, West N, Sebag-Montefiore D, et al. A prospective phase II study of pre-operative chemotherapy then short-course radiotherapy for high risk rectal cancer: COPERNICUS. $\mathrm{Br} J$ Cancer. 2018;119(6):697-706.

56. Nilsson PJ, van Etten B, Hospers GA, et al. Short-course radiotherapy followed by neo-adjuvant chemotherapy in locally advanced rectal cancer-the RAPIDO trial. BMC Cancer. 2013;13:279.

57. Grayling MJ, Dimairo M, Mander AP, Jaki TF. A review of perspectives on the use of randomization in phase II oncology trials. J Natl Cancer Inst. 2019;111(12):1255-62. https://doi.org/ 10.1093/jnci/djz126.

58. Burke DL, Billingham LJ, Girling AJ, Riley RD. Meta-analysis of randomized phase II trials to inform subsequent phase III decisions. Trials. 2014;15:346.

59. Liang F, Wu Z, Mo M, et al. Comparison of treatment effect from randomised controlled phase II trials and subsequent phase III trials using identical regimens in the same treatment setting. Eur J Cancer. 2019;121:19-28.

60. Rothwell PM. External validity of randomised controlled trials: "to whom do the results of this trial apply?". Lancet. 2005;365(9453):82-93.

61. Taylor FG, Quirke P, Heald RJ, et al. Preoperative magnetic resonance imaging assessment of circumferential resection margin predicts disease-free survival and local recurrence: 5-year follow-up results of the MERCURY study. J Clin Oncol. 2014;32(1):34-43.

62. Chetty R, Gill P, Govender D, et al. International study group on rectal cancer regression grading: interobserver variability with commonly used regression grading systems. Hum Pathol. 2012;43(11):1917-23.

63. Langer R, Becker K. Tumor regression grading of gastrointestinal cancers after neoadjuvant therapy. Virchows Arch. 2018;472(2):175-86.

64. Lau J, Ioannidis JP, Schmid CH. Summing up evidence: one answer is not always enough. Lancet. 1998;351(9096):123-7.

65. Ryan EJ, O'Sullivan DP, Kelly ME, et al. Meta-analysis of the effect of extending the interval after long-course chemoradiotherapy before surgery in locally advanced rectal cancer. $\mathrm{Br} \mathrm{J}$ Surg. 2019;106(10):1298-310. https://doi.org/10.1002/bjs.11220.

66. Sloothaak DA, Geijsen DE, van Leersum NJ, et al. Optimal time interval between neoadjuvant chemoradiotherapy and surgery for rectal cancer. Br J Surg. 2013;100(7):933-9.

67. Relton C, Torgerson D, O'Cathain A, Nicholl J. Rethinking pragmatic randomised controlled trials: introducing the "cohort multiple randomised controlled trial" design. BMJ. 2010;340:c1066.

68. Burbach JP, Kurk SA, Coebergh van den Braak RR, et al. Prospective Dutch colorectal cancer cohort: an infrastructure for long-term observational, prognostic, predictive and (randomized) intervention research. Acta Oncol. 2016;55(11):1273-80.

69. van der Velden JM, Verkooijen HM, Young-Afat DA, et al. The cohort multiple randomized controlled trial design: a valid and efficient alternative to pragmatic trials? Int $J$ Epidemiol. 2017;46(1):96-102.

Publisher's Note Springer Nature remains neutral with regard to jurisdictional claims in published maps and institutional affiliations. 